\title{
PRÉSENCE D’ANNA MARIA BATTISTA
}

\author{
Anna Maria LazZarino Del Grosso*
}

$\mathrm{I}^{1}$ l y a vingt-et-un ans, le 15 février 1988, Anna Maria Battista nous quittait prématurément. Tout en ayant dépassé la cinquantaine, elle gardait une surprenante jeunesse physique et intellectuelle. Son image rayonnante est doucement gravée dans le souvenir de ses amis. Elle était alors, et depuis longtemps, au sommet de sa carrière académique et de sa créativité scientifique. Le nombre des initiatives éditoriales et des hommages réalisés en Italie, au cours des mois et des années suivants ${ }^{1}$, témoigne de l'extraordinaire estime, de l'affection et de la reconnaissance qu'elle avait su conquérir, parmi ses élèves et ses collègues, par sa personnalité généreuse, par son dévouement passionné aux études et à l'enseignement de l'histoire de la pensée politique. Grâce à ces semailles fécondes, au cours de ces vingt dernières années, de nouvelles générations d'étudiants et de professeurs ont pu aisément connaître et apprécier ses écrits novateurs, toujours actuels. Ils en ont tiré des connaissances précieuses sur la pensée politique de l'époque moderne et contemporaine. Cet héritage révèle un parcours de recherche exemplaire par sa cohérence. Durant la trentaine d'années correspondant à l'activité et à l'engagement scientifique d'Anna Maria Battista, la France est restée le lieu privilégié de son intérêt et de ses recherches infatigables. Ce parcours, axé sur la France, acquit rapidement une importance européenne: la voie originale tracée par Anna Maria Battista présente diverses ramifications qui s'étendent à l'Italie et à l'Angleterre. Cette voie mène, presque sans interruption, de Machiavel et du problème de sa réception au $\mathrm{XVI}^{\mathrm{e}}$ siècle, jusqu'à Tocqueville et à la maturation de son chef d'œuvre: dans ses Studi tocquevilliani, paru en 1989, Anna Maria Battista analyse l'évolution, le passage de la première à la deuxième partie de La Démocratie en Amérique.

Les étapes intermédiaires de ce parcours sont évidemment nombreuses: l'antimachiavélisme des huguenots et des ligueurs, que l'on trouvera si originalement analysé

* Anna Maria Lazzarino Del Grosso, née en 1943, est professeur d'histoire des doctrines politiques à l'université de Gênes (Dipartimento di Scienze politiche e sociali, Largo Zecca 8-16, I-16124 Genova; AnnaMaria.Delgrosso@unige.it). Ses travaux portent essentiellement sur l'histoire de la pensée politique médiévale et moderne. Elle a notamment publié : Società e potere nella Germania del XII secolo. Gerhoch di Reichersberg (Florence, Olschki, 1974), Bodin e la critica della democrazia (Naples, Istituto Universitario Suor Orsola Benincasa, 2004). Le recueil de textes d'Anna Maria Battista, Politica e morale nella Francia dell'età moderna (Name, Gênes, 1998) est paru sous sa direction.

1. Voir infra, page 499, les publications posthumes d'Anna Maria Battista (parues à partir de 1989 ) et les études et les hommages qui lui sont consacrés. Dans les notes, nous indiquons les titres de ses articles et de ses ouvrages, suivis de leur année de parution. Le lecteur trouvera le détail des références dans la bibliographie finale. 
et commenté dans l'article publié en français ci-après ${ }^{2}$; la réflexion de Montaigne et de Charron, à l'égard de la politique et du pouvoir, caractérisée par une attitude critique nouvelle et l'exaltation de l'individualité privée ${ }^{3}$; la pensée hétérodoxe des libertins et des moralistes français du XVII ${ }^{\mathrm{e}}$ siècle qui, malgré leurs différences, développent une même ligne critique jusqu'au revirement apporté par les réflexions de Pierre Nicole et de Pierre Bayle permettant de valoriser, d'un point de vue exclusivement utilitaire, les actions sociales individuelles ${ }^{4}$; l'influence exercée en Angleterre par ces nouveaux enseignements, notamment sur Thomas Hobbes, avec un intérêt particulier pour la psychologie « politique » qui joua un rôle fondamental dans l'élaboration de sa théorie absolutiste $^{5}$; l'historiographie de la première moitié du XVIII ${ }^{\mathrm{e}}$ siècle et les usages idéologiques de la Germanie de Tacite ${ }^{6}$; l'étude de Rousseau et de Robespierre, s'opposant à l'individualisme utilitaire, justifiant l'absolutisme, affirmant la supériorité de l'intérêt publique et l'exaltation de la morale communautaire ${ }^{7}$; l'analyse de la réception des idées politiques de Rousseau chez les principaux représentants du jacobinisme ${ }^{8}$ ainsi que dans le projet constitutionnel de Bolívar?.

Les nombreux écrits dédiés par Anna Maria Battista, durant une quinzaine d'années, à un commentaire savamment novateur de La Démocratie en Amérique de Tocqueville ${ }^{10}$ marquent la dernière étape de ce cheminement historiographique. Sa logique intrinsèque, fruit d'une exigence progressive d'éclaircissements à l'intérieur d'un même cadre thématique, se superpose à la stricte chronologie. La crainte d'un retour au jacobinisme et aux manifestations extrêmes de la souveraineté populaire anime, d'après la lecture d'Anna Maria Battista, la "première » Démocratie en Amérique. Le jacobinisme est donc l'un des liens qui unissent cette veine dixneuviémiste au reste du corpus des études battistiennes consacrées à la pensée politique moderne. La mort de l'auteur en a fait, hélas, la ligne d'arrivée, le point final de son œuvre. On peut supposer, pourtant, que son avancée dans l'histoire contemporaine n'était pas destinée à s'arrêter là. Sa production fournit, dans son ensemble, un vaste matériau pour la rédaction d'un puissant livre de synthèse sur la pensée politique française du XVI ${ }^{\mathrm{e}}$ au $\mathrm{XIX}^{\mathrm{e}}$ siècle. Ce grand livre, d'ailleurs, existe déjà : les lecteurs attentifs saisiront sans peine son contenu, en relisant la totalité de ses travaux, les uns après les autres, en suivant leur chronologie.

2. Voir infra, p. 501-531. Il s'agit de la traduction française de « Sull'antimachiavelismo francese del secolo XVI », 1962.

3. Alle origini del pensiero politico libertino. Montaigne e Charron, 1966.

4. «Appunti sulla crisi della morale comunitaria del Seicento francese », 1969; « Morale "privata" e utilitarismo politico nella Francia del Seicento », 1974; « Morale privée et utilitarisme politique en France au XVII ${ }^{\mathrm{e}}$ siècle », 1975; "Come giudicano la "politica" libertini e moralisti nella Francia del Seicento », 1980; Nascita della psicologia politica, 1982.

5. Nascita della psicologia politica, 1982.

6. «La Germania di Tacito nella Francia illuminista », 1979.

7. « Il "Rousseau" dei giacobini », 1977; I Principi della democrazia, 1983.

8. Voir Il « Rousseau » dei giacobini. Studi in onore di Anna Maria Battista, 1988.

9. «El poder moral : la creazione irrisolta e sconfitta di Simon Bolívar», 1987.

10. «Lo "Stato Sociale Democratico" nella analisi di Tocqueville e nelle valutazioni dei contemporanei », 1973; Lo Spirito liberale e lo spirito religioso. Tocqueville nel dibattito sulla scuola, 1976; « Il primo Tocqueville sulla "Democrazia politica" », 1981; " Tocqueville. Un tentativo di sintesi », 1985 ; Studi tocquevilliani, 1989. 
En 1956, après sa maîtrise en sciences politiques à l'université de La Sapienza à Rome, Anna Maria Battista y devint successivement l'élève et l'assistante de Rodolfo De Mattei qui était alors titulaire de la chaire d'Histoire des doctrines politiques ${ }^{11}$. C'est à ce guide qu'elle doit d'avoir été initiée à la méthode historique, fondée en premier lieu sur la philologie la plus rigoureuse et sur la prise en compte de tout type de sources touchant aux idées politiques, y compris les plus négligées. La fréquentation, l'estime et l'amitié de Luigi Firpo confirmeront au fil du temps la fécondité de ce choix. Son premier article, «La penetrazione del Machiavelli in Francia nel secolo XVI », paru en 1960, montre que la leçon du maître fut promptement assimilée. S'appuyant sur une culture historique remarquable, Anna Maria Battista saisissait déjà les grands problèmes interprétatifs posés par les sujets qu'elle traitait, corrigeant ou bouleversant des positions historiographiques établies en leur opposant une série de sources jusque-là ignorées ou mal connues. Il s'agissait, dans son premier article, des traductions françaises des Discours, du Prince et de L'Art de la guerre de Machiavel, parues entre 1544 et la fin du siècle. Leur analyse lui permit de mettre en évidence le bon accueil réservé au secrétaire florentin par l'entourage de la reine mère: elle démontrait que ces écrits de Machiavel étaient essentiellement appréciés pour leur enseignement pratique. De ce point de vue, l'incompatibilité de leur « réalisme politique » avec la doctrine aristotélicienne et thomiste, postulant une liaison indissoluble entre morale et politique, semblait donc secondaire. Considéré superficiellement comme un ouvrage à l'appui de l'idéologie monarchiste, le Prince était lié, selon Anna Maria Battista, à la figure de Catherine de Médicis telle que dépeinte par les huguenots: la responsabilité du massacre de la Saint-Barthélemy avait été imputée à la reine mère qui aurait suivi, en cela, les maximes politiques du Florentin. Les huguenots, assimilant Le Prince à un manuel à l'usage des tyrans, ne pouvaient en acquérir une connaissance et une compréhension satisfaisantes, fidèles à son contenu.

Ce point est clairement démontré par une série de travaux qui porte sur la fortune de Machiavel dans la France du $\mathrm{XVI}^{\mathrm{e}}{ }$ siècle ${ }^{12}$, en particulier dans son article consacré à l'antimachiavélisme français de l'époque $^{13}$. Ce texte éclaire la différence entre l'antimachiavélisme des huguenots et celui des ligueurs, mais aussi leur point commun: l'ignorance et la trahison de la pensée de Machiavel. Anna Maria Battista achève ainsi sa destruction du mythe historiographique du clivage causé dans la pensée politique française par la diffusion des œuvres de Machiavel; ce faisant, elle remet radicalement en cause la thèse de leur influence capitale dans le processus de transition vers la « modernité ». En poursuivant ses recherches sur la réception française de Machiavel par l'analyse de Montaigne ${ }^{14}$, elle souligne que les Essais témoignent d'un manque d'intérêt pour la science politique enseignée dans Le Prince et pour son auteur qui

11. Sur Rodolfo De Mattei voir Luciano Russi, Il Passato del presente. Rodolfo De Mattei e la storia delle dottrine politiche in Italia, Pescara, Edizioni Scientifiche Abruzzesi, 2005.

12. Dans « Pasquier e Machiavelli », 1961, par exemple, elle avait montré, en passant minutieusement au crible les écrits de l'auteur des Recherches de la France, l'authenticité de son antimachiavélisme, discutée par Vittorio De Caprariis quelques années auparavant (voir Vittorio DE CAPRARIIS, Propaganda e pensiero politico in Francia durante le guerre di religione, Naples, Edizioni scientifiche italiane, 1959). Selon Battista, Pasquier ne voyait dans Le Prince qu'un recueil de mauvais préceptes longuement pratiqués dans l'histoire.

13. « Sull’antimachiavellismo francese del secolo XVI», 1962.

14. « Montaigne e Machiavelli », 1963. 
restait, d'ailleurs, attaché à une vision humaniste de la politique alors conçue comme le seul domaine où les hommes pouvaient réaliser leurs buts et leurs espoirs. Les recherches d'Anna Maria Battista nous font percevoir à quel point Montaigne refusa cette vision, en choisissant la voie tout à fait novatrice et intellectuellement révolutionnaire de la «solitude», du détachement critique de toute valeur sociale reçue comme de toute institution politique. Dans sa communication, présentée au congrès de Florence pour le $\mathrm{V}^{\mathrm{e}}$ centenaire de la naissance de Machiavel ${ }^{15}$, elle revient de façon magistrale sur sa thèse du déclin de l'intérêt pour Machiavel dans la France des dernières années $\mathrm{du} \mathrm{XVI}{ }^{\mathrm{e}}$ siècle. Chez les penseurs les plus subtils et les plus inquiets, la foi dans les valeurs civiques avait été détruite par l'expérience tragique des guerres de Religion. Ce déclin se poursuit au cours du XVII ${ }^{\mathrm{e}}$ siècle, lorsque l'attention pour les ouvrages du Florentin ne mène qu'à l'évocation de préceptes « machiavéliques » dans les milieux courtisans, tandis que chez les esprits les plus éclairés triomphent le refus de l'engagement politique et la recherche d'un bonheur individuel.

Son livre de 1966, Alle origini del pensiero politico libertino. Montaigne e Charron, marque un tournant décisif dans son parcours scientifique. Elle y démontrait que les accusations de machiavélisme adressées à Montaigne et à Charron consistaient en une marque d'infamie utilisée par les champions de l'orthodoxie religieuse des deux confessions, catholique et protestante, dans le but de condamner indistinctement tous les écrivains dont les opinions politiques paraissaient hétérodoxes. En analysant les œuvres de ces deux auteurs, elle en renouvela la lecture: elle affirma, d'une part, l'existence d'une pensée politique propre à Montaigne, dont l'influence sur Charron fut fondamentale et féconde; elle démontra, d'autre part, que cette pensée politique, parvenue de manière autonome à séparer les domaines de la morale et de la politique, constitue l'origine de la révolution intellectuelle représentée par le passage à la modernité politique. Ce livre sur Montaigne et Charron assoit la renommée scientifique de son auteur. L'année même de sa parution, Anna Maria Battista débute son enseignement de l'histoire des doctrines politiques à l'université d'Urbino. Au début des années 1970, une "école » formée par ses élèves commence à émerger. Nombreux enseigneront à leur tour à l'université, dans différentes villes de la péninsule. En 1974, elle conçoit le projet d'une recherche collective sur l'image de Rousseau telle qu'elle a été façonnée par les principaux représentants du jacobinisme, confiant à chacun de ses élèves la tâche particulière d'étudier l'un d'eux. À partir de 1975, elle poursuit son enseignement à Rome, à l'université de La Sapienza: le souvenir partagé de l'expérience heureuse, et presque mythique, d'une communauté intellectuelle très vivante, parfois rieuse, demeure. Cette communauté, dont elle était le guide, constituait le lieu où étaient discutés les résultats de ses « trouvailles » parisiennes qui alimentèrent ses écrits durant les années 1969-1980: ces textes consacrés aux libertins et aux moralistes, examinant la dissociation entre morale et politique comme la primauté des buts et des valeurs privés qu'ils faisaient valoir. Après son départ pour Rome, et jusqu'à sa mort, elle reviendra très souvent à Urbino, ville qu'elle chérissait et qu'elle appelait « le royaume enchanté de [sa] jeunesse ${ }^{16}$ ».

15. «Direzioni di ricerca per una storia di Machiavelli in Francia », 1972.

16. Voir la note préliminaire de $I l$ «Rousseau » dei giacobini. Studi in onore di Anna Maria Battista, 1988, p. 7. 
Un congrès sur la fortune de Tacite, organisé à Urbino au mois d'octobre 1978, fut pour elle l'occasion de réfléchir sur la fortune de la Germanie. Elle entama ainsi ses recherches dans le domaine de l'historiographie politique française du XVIII' siècle à travers Vertot, Boulainvilliers, Dubos, Montesquieu et Mably.

Durant ses années romaines, avec ses collègues et amis Luigi Firpo, Nicola Matteucci, Salvo Mastellone, Arduino Agnelli, elle participa à la revue Il Pensiero Politico, fondée en 1968. Par son dynamisme, elle contribua largement à la formation du milieu intellectuel qui créa en 1995, à Florence, l'Associazione italiana degli storici delle dottrine politiche. Sa belle maison, située face à l'île Tibérine, était le point de convergence d'un grand nombre d'amis, de collègues, de jeunes chercheurs. Par son accueil cordial et généreux, elle distribuait abondamment les fruits de ses connaissances, communiquait son enthousiasme et émerveillait par l'exemple: la rigueur qui accompagnait son humilité, le besoin de revenir constamment sur ses pas avant de définir de nouveaux objectifs de recherche, les conseils qu'elle prodiguait, le sens des défis intellectuels qu'elle savait transmettre restent dans notre mémoire.

Dans son dernier article, "Nuove riflessioni su "Montaigne politico" ", écrit en l'honneur de Luigi Firpo, elle revenait à Montaigne. Contredisant Burckhardt, qui avait placé dans la Renaissance l'origine de l'individualisme moderne, elle y soutenait, par une argumentation limpide et serrée, l'attribution à Montaigne de cette paternité novatrice. Sa dédicace à l'ami révéré exprime de manière suggestive le modèle qui a orienté son existence de chercheuse, marquée par son goût pour l'histoire des grands mouvements intellectuels de l'âge moderne et par une sensibilité particulière au problème qui hante tous ses écrits, celui des rapports entre morale et politique: " Il me plaît d'offrir à Luigi Firpo, qui nous a enseigné que la recherche est une histoire sans repos et sans fin, cet essai concernant un sujet qui m'est cher - le Montaigne politique - auquel je reviens après bien des années dans une version nouvelle qui, je l'espère, ne sera pas la dernière ${ }^{17}$. » Revenir aujourd'hui à l'œuvre d'Anna Maria Battista permet de réfléchir sur la valeur de ce modèle, d'en goûter les fruits. La richesse de cette œuvre passionnée ne cesse d'exercer un charme fécond sur ses nouveaux lecteurs ${ }^{18}$.

17. « Nuove riflessioni su "Montaigne politico" », 1990, p. 801.

18. Nous remercions Laurie Catteeuw pour son aide précieuse dans la rédaction de ce texte et pour son initiative qui vise à rendre plus accessibles les travaux d'Anna Maria Battista aux lecteurs français, comme en témoigne la publication de la traduction ci-après (p. 501-531). Bon nombre de ses travaux ont vocation à être reçu dans la patrie de Montaigne. 


\section{BIBLIOGRAPHIE D'ANNA MARIA BATTISTA}

1960 «La penetrazione del Machiavelli in Francia nel secolo XVI», Rassegna di politica e storia, vol. VII, p. 1-16 (*27-51) ${ }^{19}$.

1961 «Pasquier e Machiavelli », Rivista internazionale di filosofia del diritto, vol. XXXVIII, p. 491-516 (*53-73).

1962 «Sull'antimachiavellismo francese del secolo XVI », Storia e politica, vol. I, p. 413-447 (*75-107).

1963 «Montaigne e Machiavelli», Rivista internazionale di filosofia del diritto, vol. XL, p. 526-563.

1966 Alle origini del pensiero politico libertino. Montaigne e Charron, Milan, Giuffré (rééd. revue et corrigée par l'auteur, publiée chez le même éditeur, en 1979).

1969 «Appunti sulla crisi della morale comunitaria del Seicento francese », Il Pensiero Politico, vol. II, p. 187-223 (*155-187).

«Sul rapporto tra società e Stato nella Francia dell'assolutismo: ancora a proposito di un saggio su Antonio Negri », Quaderni storici delle Marche, vol. IV, p. 85-113 $(* 137-153)$.

1972 «Direzioni di ricerca per una storia di Machiavelli in Francia », dans Il Pensiero politico di Machiavelli e la sua fortuna nel mondo, Atti del Convegno per il V centenario della nascita di N. Machiavelli (S. Casciano/Firenze, 28-29 settembre 1969), Florence, Istituto nazionale di studi sul Rinascimento, p. 37-66; rééd. dans La Cultura, vol. X, 1972, p. 186-218 (*109-135). Deux ans auparavant, l'auteur avait publié ce texte, avec un paragraphe introductif supplémentaire, sous forme d'opuscule (Rome, Abete, 1970).

1973 «Lo "Stato Sociale Democratico" nella analisi di Tocqueville e nelle valutazioni dei contemporanei », Il Pensiero Politico, vol. VI, p. 336-395 (**65-145).

1974 «Morale "privata” e utilitarismo politico nella Francia del Seicento », Storia e politica, vol. XIII, p. 507-545 (*189-219).

1975 « Morale privée et utilitarisme politique en France au XVII siècle », dans Roman SCHNUR, dir., Staatsräson. Studien zur Geschichte eines politischen Begriffs, Internationales Kolloquium über Staatsräson, Tübingen, 2-5 April 1974, Berlin, Duncker \& Humblot, p. 87-119.

1976 Lo Spirito liberale e lo spirito religioso. Tocqueville nel dibattito sulla scuola, Milan, Jaca Book.

1977 «Il "Rousseau" dei giacobini », Trimestre, vol. X, p. 3-22.

1979 «La Germania di Tacito nella Francia illuminista », dans Franco GoRI et Cesare CuEstA, dir., La Fortuna di Tacito dal sec. XV ad oggi, Atti del colloquio di Urbino, 9-11 ottobre 1978 (numéro spécial des Studi Urbinati, vol. LIII), p. 93-131; rééd. sous forme d'ouvrage avec une préface de Diego Quaglioni (Urbino, Edizioni Quattroventi, 1999).

1980 «Come giudicano la "politica" libertini e moralisti nella Francia del Seicento », dans Sergio Moravia, éd., Il Libertinismo in Europa, Milan/Naples, Ricciardi, p. 25-80.

19. Dans la bibliographie, les références suivies d'un astérisque ont été rééditées dans Politica e morale nella Francia dell'età moderna (Gênes, Name, 1998); celles qui sont suivies de deux astérisques, dans I Principi della democrazia (Chieti, Trimestre Editore, 1989). Les chiffres indiqués entre parenthèses correspondent aux pages des articles concernés dans l'un ou l'autre de ces ouvrages. 
1981 « Il primo Tocqueville sulla "Democrazia politica" (in margine a un saggio recente)», Quaderni fiorentini per la storia del pensiero giuridico moderno, vol. X, p. 9-52 (**146191).

1982 Nascita della psicologia politica, prefazione Anna Maria Lazzarino Del Grosso, Gênes, ECIG (Edizioni Culturali Internazionali Genova).

1983 Maximilien de RoBespierre, I Principi della democrazia. Raccolta di discorsi svolti all'Assemblea Costituente francese, 1789-1791, éd. Anna Maria Batтista, Pescara, Clua Editrice; rééd. sous la dir. de Luciano Russi, Chieti, Trimestre Editrice, 1989, puis Padoue, CEDAM, 1997.

1985 « Tocqueville. Un tentativo di sintesi », Trimestre, vol. XVII, p. 171-244 (**192-268).

1987 «El poder moral: la creazione irrisolta e sconfitta di Simon Bolívar », Il Pensiero Politico, vol. XX, p. 56-78.

1989 Studi tocquevilliani, introduzione Francesco De Sanctis, appendice Ettore Cuomo, Florence, Centro editoriale toscano.

1990 «Nuove riflessioni su "Montaigne politico"», dans Silvia Rota GHibaudi et Franco BARCIA, éd., Studi politici in onore di Luigi Firpo, vol. I, Milan, Angeli, p. 801-848.

L'Assolutismo laico, éd. Anna Maria Battista, introduzione Lucio Pala, traduzione e note Diana THERMES, Milan, Giuffré.

1997 « Il dibattito politico nell'Italia della Controriforma: ragion di Stato, tacitismo, machiavellismo, utopia » (en collab. avec Artemio Enzo BALDINI), Il Pensiero Politico, $\mathrm{n}^{\circ} 3$, p. 393-409.

1998 Politica e morale nella Francia dell'età moderna, a cura di Anna Maria LAzZARINo Del Grosso, Gênes, Name.

\section{ÉTUDES ET HOMMAGES}

1988 Il «Rousseau » dei giacobini. Studi in onore di Anna Maria Battista, éd. Franco Barcia, Bruna Consarelli, Marinella Fedeli De Cecco et Lucio Pala, Pubblicazioni dell’Università degli Studi di Urbino, Urbino, Edizioni Quattro Venti.

1989 Dal machiavellismo al libertinismo. Studi in memoria di Anna Maria Battista, numéro spécial de la revue Il Pensiero Politico (XXII/2). Le volume réunit les textes des relations présentées à Florence le 22 octobre 1988.

Studi in onore di Anna Maria Battista. Dalla Rivoluzione alla Restaurazione alla Rivoluzione, numéro spécial de la revue Trimestre (XXII/2-3-4).

1992 Individualismo. Assolutismo. Democrazia, éd. Vittorio Dini et Domenico Taranto, Edizioni Scientifiche Italiane (Pubblicazioni dell'Università degli Studi di Salerno, Sezione Atti, convegni, miscellanee, $\mathrm{n}^{\circ} 34$ ). Il s'agit des actes du colloque tenu en l'honneur d'Anna Maria Battista, à Salerne, du 12 au 14 octobre 1989.

«Inaugurazione del Dipartimento di Storia e critica della politica (Teramo 22-23 marzo 1991) », Universita' degli studi Gabriele d'Annunzio, Dipartimento di storia e critica della politica, Anno accademico 1991-1992, p. 21-43. Ce texte est une présentation du volume Studi in onore di Anna Maria Battista. Dalla Rivoluzione alla Restaurazione alla Rivoluzione paru en 1989 (op. cit.).

1993 Anna Maria Battista. Ricordi e testimonianze, Terracina, Casa Editrice Gabbiano (Centro di Studi Mediterranei). 